\title{
Precise Preoperative Localization of Prostate Cancer Employing 12-Core Needle Biopsy with a Tissue-Marking Method for Effective Surgical Strategy
}

\author{
Satoko Sato, ${ }^{1}$ Mika Watanabe, ${ }^{1}$ Shinji Taniuchi, ${ }^{1}$ Atsuko Kasajima, ${ }^{1}$ \\ Koji Mitsuzuka, ${ }^{3}$ Yasuhiro Nakamura, ${ }^{2}$ Fumiyoshi Fujishima, ${ }^{1}$ Yoichi Arai ${ }^{3}$ \\ and Hironobu Sasano ${ }^{1,2}$ \\ ${ }^{1}$ Department of Pathology, Tohoku University Hospital, Sendai, Miyagi, Japan \\ ${ }^{2}$ Department of Anatomic Pathology, Tohoku University Graduate School of Medicine, Sendai, Miyagi, Japan \\ ${ }^{3}$ Department of Urology, Tohoku University School of Medicine, Sendai, Miyagi, Japan
}

\begin{abstract}
Prostate needle biopsy plays a pivotal role not only in the diagnosis but also the management of patients with prostate cancer. Prostate cancer is often multifocal and diagnosis of the lesion could therefore be difficult with diagnostic imaging only; thus, multiple core biopsies are taken from several different regions of the prostate. In current practice, 10- or 12-core needle biopsy is considered the clinical standard. Several techniques have been reported to improve the orientation of the specimens, but tissue marking, which could theoretically provide important information on the location of the lesion in the prostate, has been rarely reported. Therefore, in this study, we evaluated the clinical significance of systematic 12-core needle biopsy with tissue marking for preoperative prediction of lesion sites and clinicopathological features of patients. We evaluated 93 patients who underwent 12-core prostate biopsy and subsequent radical prostatectomy. We correlated the biopsy results to the prostate sites in which biopsies were performed and prognostic factors of the patients, especially the degree of extraprostatic extension (EPE) obtained in surgical specimens. Among 253 cancer foci detected in 93 prostatectomy specimens, 168 (66.4\%) foci were detected by biopsy. All patients had proven cancer. EPE-positive cancers were associated with a larger number of positive cores, larger tumor length, and higher percentage of cancer tissue in the corresponding cores. Systematic 12-core prostate biopsy with tissue marking is useful for preoperative detection of cancer foci and provides valuable information that enables effective surgical strategies.
\end{abstract}

Keywords: biopsy; extraprostatic extension; histopathology; multifocality; tissue marking

Tohoku J. Exp. Med., 2015 May, 236 (1), 55-61. (C) 2015 Tohoku University Medical Press

\section{Introduction}

The prevalence of prostate cancer has markedly increased in Japan over the past decades. This increased incidence could be partly explained by the widely applied prostate-specific antigen (PSA) screening (Sonoda et al. 2004). Needle biopsy is the most common diagnostic modality for prostate cancer after serum PSA screening. Prostate cancers are often multifocal and, in contrast to other human malignancies, its diagnosis can be very challenging when only diagnostic imaging such as ultrasonography or magnetic resonance imaging (MRI) is used. Therefore, prostate biopsies are taken from several regions of the prostate at this juncture. Because of the diagnostic importance of prostate biopsy sampling, various biopsy procedures have been developed. Currently, sampling by a 10 - or 12-core needle at the lateral zones is considered the standard practice (Eskew et al. 1997; Babaian et al. 2000).

When a histopathological diagnosis of cancer is determined by needle biopsy, treatment algorithms including surgical procedures are determined according to the localization of the cancer, histological grade, and life expectancy of patients. Most prostate cancers are acinar adenocarcinomas, and other types of adenocarcinoma such as ductal adenocarcinoma and mucinous carcinoma are rare. The histological grade of prostate cancer is usually reported according to the Gleason scoring system (Bostwick 1994). The Gleason score is calculated on the basis of the dominant histological grades, from grade 1 (well differentiated) to grade 5 (poorly differentiated). The score is derived by adding the two most prevalent pattern grades and is often indicated by its separate components (e.g., Gleason score 4 $+3=7$ ). Prostate needle biopsy is useful for the management of prostate cancer and also for predicting pathological

Received September 24, 2014; revised and accepted April 23, 2015. Published online May 14, 2015; doi: 10.1620/tjem.236.55.

Correspondence: Satoko Sato, M.D., Ph.D., Department of Pathology, Tohoku University Hospital, 1-1 Seiryo-machi, Aoba-ku, Sendai, Miyagi 980-8574, Japan. e-mail: satoshio@patholo2.med.tohoku.ac.jp 
features of radical prostatectomy (Bjurlin et al. 2013). Histopathological findings obtained by prostate core needle biopsy, including the number of cancer-positive cores, the length of the cancer, the percentage of cancer tissue in the cores sampled, and the Gleason score, have all been reported to be associated with various prognostic factors, including tumor size, the presence of extraprostatic extension (EPE), positive surgical margin, seminal vesicle invasion, and perineural invasion (pn) (Pound et al. 1999; Stamey et al. 1999; Quinn et al. 2003). However, little is known about the association of biopsy results with multifocality_and anatomical localization of prostate cancer (Bjurlin et al. 2013).

Tissue marking techniques for prostate biopsy specimens have been reported in some studies (Terris and McNeal 2002; Firoozi et al. 2009). However, in these studies, tissue markings were performed to distinguish each needle biopsy core, not necessarily identifying the orientation of each core, and its advantages of predicting cancer location was therefore rather limited. Recently, the orientation of each biopsy core, with or without the use of tissue marking techniques, has been attempted, and its benefits in predicting a more precise location of the foci of cancer have been reported in the literature (Galosi et al. 2011; Yamanaka et al. 2013). However, these works did not provide the details regarding cancer localization. Therefore, the precise localization of the intraprostate sites of the cancer is pivotal in determining the treatment algorithm of the patients; however, no studies are available in this field.

Therefore, in this study, we examined whether the 12-core needle biopsy with the newly developed tissue marking technique could enable us to identify the location, multifocality, and EPE of prostate cancer in a more precise manner compared with the results obtained from surgical specimens of prostate cancer.

\section{Materials and Methods}

In this study, 93 patients with prostatic acinar adenocarcinoma were evaluated, all of which were diagnosed by initial prostate core needle biopsy from 12 portions (six from the right side and six from the left side). All patients subsequently received total prostatectomy at Tohoku University Hospital, Sendai, Japan between 2003 and 2012. Patients diagnosed by repeated biopsy and preoperative hormonal therapy and/or irradiation were excluded from the study.

In all patients, prostate needle biopsies were performed transrectally using the ALOKA Pro Sound 5000 (Aloka, Tokyo, Japan) with a $5-\mathrm{MHz}$ endocavitary probe and an 18-gauge core biopsy needle with a spring-loaded biopsy gun. Biopsies were systematically sampled from 12 portions as follows: three from the medial part, two from the lateral part, in each of the lobes, and two from the anterior apical peripheral zone (Orikasa et al. 2008) (Fig. 1A). Each biopsy was marked with black ink at the anterior end (opposite site of the rectal side) following sampling by urologists prior to fixation in $10 \%$ formalin. This method makes it possible to identify cancer localization. Each sample was fixed in $10 \%$ buffered formalin, embedded in paraffin, and subsequently sectioned in $2-\mu \mathrm{m}$ thick slices. Tissue sections were stained with hematoxylin and eosin (HE). HE-stained specimens were microscopically examined to calculate Gleason score, length and percentage of tumor tissue, and localization (\{posterior [rectal (capsular) side], middle, or anterior [opposite rectal (capsular) side]\}) of the cancer portion.

All prostatectomy specimens were fixed in $10 \%$ buffered formalin for 1-2 days. Fixed specimens were also marked with ink over the entire surface and cut at 5-mm intervals from the apex to the base. These transversely resected segments were submitted for wholemount processing. Cancer foci were considered different if they were separated by more than $5 \mathrm{~mm}$ in a single section or if they were located in a different region from the cancer lesion of the adjacent (above or below) section (Fig. 1B). The histopathological features of each cancer lesion were individually and carefully evaluated. The parameters assessed for each surgical specimen included tumor size, localization, Gleason score, pn, EPE, and resection margin (RM).

The detectability of foci of adenocarcinoma in biopsy specimens was assessed retrospectively by comparing the histopathological features of prostatectomy and biopsy specimens. Individual cancer lesions in the prostatectomy specimens were defined as detectable in the biopsy (a detected cancer) if one or more biopsy cores corresponding to its location were positive for cancer. Statistical analysis was performed using the chi-square test.

Because one of the most important factors determining the clinical stage of prostate cancer is EPE, we also evaluated whether we could determine the presence of EPE in the biopsy specimens in conjunction with preoperative serum PSA levels and histological parameters using univariate and multivariate logistic regression analyses. Histological parameters included number of positive cores, the Gleason score, maximum tumor length, and the maximum percentage of cancer tissue in the most affected core.

All statistical analyses were performed using JMP Pro version 10.0.2 (SAS Institute Inc., Cary, NC, USA). A $P$-value $<0.05$ was considered statistically significant.

The Ethics Committee at Tohoku University School of Medicine approved the research protocols of this study.

\section{Results}

Table 1 summarizes the clinicopathological features of the 93 patients evaluated in the study. The mean age of the patients was $64.2 \pm 6.0$ years. The mean length of biopsy specimens was $15.1 \pm 0.1 \mathrm{~mm}$. The mean number of cancer-positive biopsy cores was $3.2 \pm 2.1$ per patient. The overall (global) Gleason score ranged from 6 to 9 and the number of patients for each score was as follows: score 6 , 39 (42\%); score 7, 47 (51\%); score 8, $5(5 \%)$; and score 9, $2(2 \%)$.

In total, 253 separate adenocarcinoma lesions were observed in the prostatectomy specimens from the 93 patients. The mean number of cancer foci per patient was $2.7 \pm 1.3$, and two or more different foci of adenocarcinoma were identified in 75 (81\%) patients. The number of cancer foci per patient was not correlated with patient age or PSA ( $P=0.93$ and $P=0.1$, respectively; data not shown). The mean maximum length of the cancer lesions was $10.1 \pm 7.5$ $\mathrm{mm}$. With regard to cancer localization, $110(44 \%)$ lesions were observed in the anterior region, $125(49 \%)$ in the posterior, and $18(7 \%)$ in both the anterior and posterior regions. In total, $189(75 \%)$ lesions were observed in the 
(A)
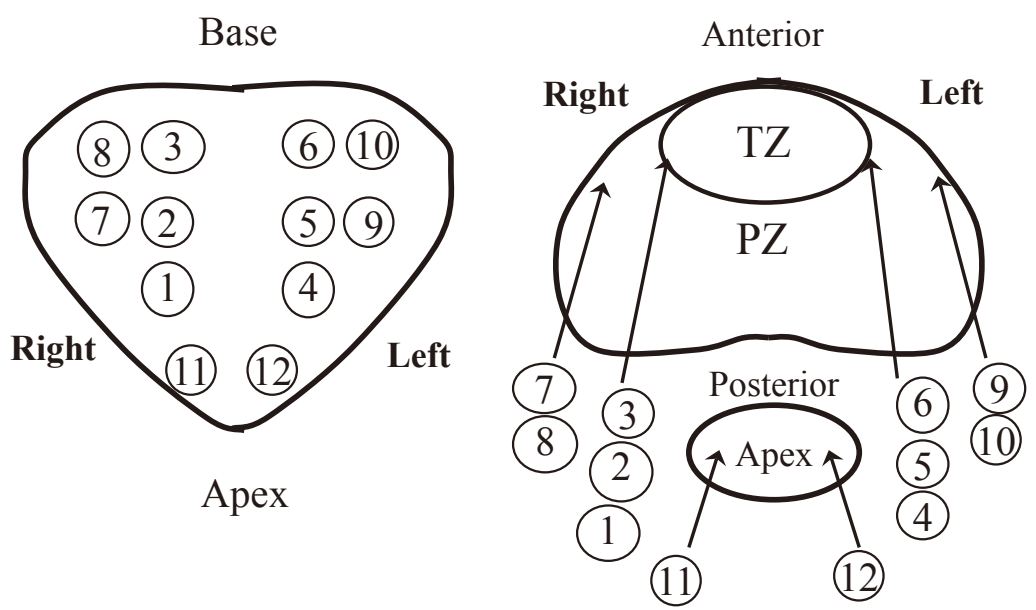

(B)

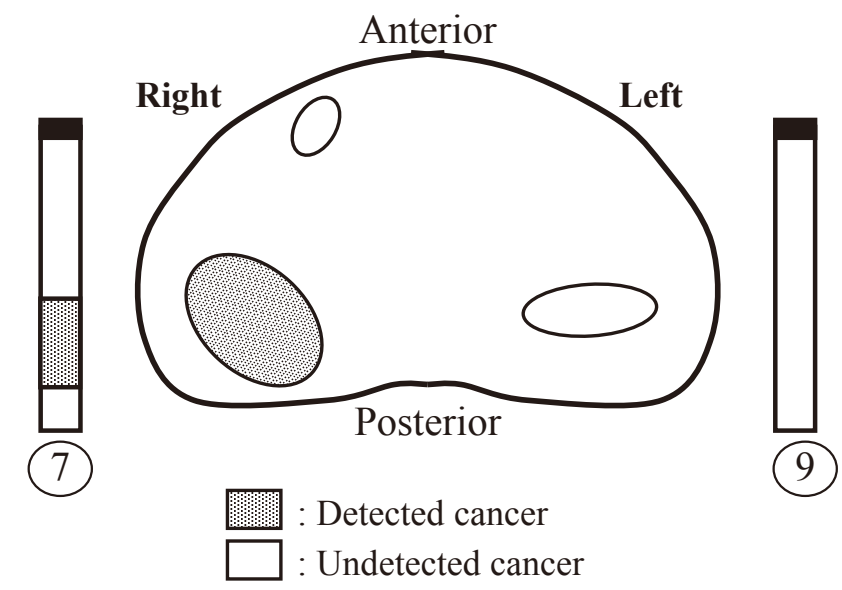

Fig. 1. Overview of the biopsy protocol and prostatectomy specimens.

(A) Representative illustration of the 12-corebiopsy protocol. Left, frontal sections; right, horizontal sections. \#1-6 correspond to sextant, \#7-\#10 to lateral biopsies, and \#11-\#12 to apical anterior peripheral zone (AAPZ) biopsies. TZ, transition zone; PZ, peripheral zone.

(B) Definition of cancer detectability. Among the three cancers illustrated in the figure, a meshed tumor was defined as detectable foci of carcinoma, including that on biopsy core of \#7.

peripheral zone (PZ), whereas $64(25 \%)$ were observed in the transitional zone (TZ). The mean maximum length of the cancer lesions was $10.1 \pm 7.4 \mathrm{~mm}$ in the $\mathrm{PZ}$ and $10.1 \pm$ $7.8 \mathrm{~mm}$ in the TZ, with no differences between the PZ and $\mathrm{TZ}$ lesions. The Gleason score of individual cancer lesions was $6,7,8$, and 9 in $131(52 \%), 115(45 \%)$, six $(2.4 \%)$, and one $(0.4 \%)$ patients, respectively. The number of foci exhibiting EPE was 15 (6\%; 14 patients), pn was 15 (6\%; 14 patients), and positive RM was 27 (11\%; 24 patients).

In total, 168/253 lesions identified in the prostatectomy specimens $(66.4 \%)$ were detected in the biopsy specimens (Table 2). The mean maximum length of detected cancers was $12.4 \pm 7.8 \mathrm{~mm}$, which were significantly larger $(P=0.0011)$ than that of undetected cancers $(5.5 \pm 3.8$ $\mathrm{mm})$. The cancer detection rate was $64.5 \%(71 / 110)$ in the anterior region, $67.2 \%(84 / 125)$ in the posterior region, and $72 \%(13 / 18)$ in both the anterior and posterior regions $(P=$
0.81). The detectability of carcinoma in the $\mathrm{PZ}$ was $68.3 \%$, showing a slightly higher rate than the detectability of carcinoma in the TZ $(60.9 \%)$, although no statistical significance was detected $(P=0.28)$. The mean maximum length of detected cancers was $12.3 \pm 7.6 \mathrm{~mm}$ in the $\mathrm{PZ}$ and $13.0 \pm$ $8.4 \mathrm{~mm}$ in TZ, with no statistically significant difference. The number of carcinoma lesions with high Gleason scores $(>7)$ was $101(60.1 \%)$ in detected cancers and $21(24.7 \%)$ in undetected cancers $(P<0.0001)$. In the detected cancers, the positive rate of EPE was $93 \%(14 / 15)$, the positive rate of pn was $90 \%(55 / 61)$, and the positive rate of RM was $100 \%(27 / 27)(P<0.0001, P=0.02, P<0.0001$, respectively). One patient had an EPE-positive cancer lesion that had not been preoperatively detected, although the same patient had another EPE-positive cancer lesion that had been identified.

Parameters related to the preoperative prostate biopsy 
correlating with the existence of EPE in the prostatectomy specimen included the number of cancer-positive biopsy cores, the mean maximum length of the biopsy core per cancer lesion, and the mean maximum percentage of cancer tissue (Table 3). The number of positive biopsy cores was significantly higher in EPE-positive tumors (mean, $2.9 \pm$ 1.4) than in EPE-negative tumors (mean, $1.8 \pm 1.4)(P=$

Table 1. Clinicopathological features of 93 patients.

\begin{tabular}{cc}
\hline & $\begin{array}{c}\text { All patients } \\
(n=93)\end{array}$ \\
\hline Mean Age, yr (range) & $64.2(49-77)$ \\
Mean serum PSA (ng/m) (range) & $6.3(1.3-17.8)$ \\
Biopsy results (range) & \\
No. of positive core & $3.2(1-10)$ \\
Max. tumor length (mm) & $4.9(0.5-14)$ \\
Max. occupation rate $(\%)$ & $33.2(2.5-100)$ \\
Overall Gleason score (\%) & \\
6 & $39(41.9)$ \\
7 & $47(50.5)$ \\
8 & $5(5.4)$ \\
9 & $2(2.2)$ \\
Prostatectomy specimen (range) & \\
No. of tumor/patient & $2.7(1-7)$ \\
No. of detected tumor & $1.8(1-4)$ \\
No. of undetected tumor & $1.0(0-4)$ \\
Max. length of cancer (mm) & $10.5(1-38)$ \\
Overall Gleason score (\%) & \\
6 & $21(22.6)$ \\
7 & $68(7.3)$ \\
8 & $1(1.0)$ \\
RM positive & $3(3.2)$ \\
\hline & $25(26.9)$ \\
& $52(55.9)$ \\
\hline &
\end{tabular}

0.01). The mean maximum percentage of cancer tissue among the corresponding biopsy cores was also significantly higher in EPE-positive cancers $(46.4 \pm 26.9 \%)$ than in EPE-negative cancers $(24.5 \pm 20.6 \%)(P=0.0003)$. The mean maximum length of the biopsy cores per cancer was $6.6 \pm 3.1 \mathrm{~mm}$ in EPE-positive cancers and $3.5 \pm 2.9 \mathrm{~mm}$ in EPE-negative cancers $(P<0.0001)$. There were 15 EPEpositive cancer lesions in the prostatectomy specimens; among these 14 foci of adenocarcinoma had been detected by the preoperative biopsy, all of which were detected in different patients. One EPE-positive cancer remained undetected in the preoperative biopsy; this patient was one of the aforementioned 14 patients, i.e., only one patient had two EPE-positive cancer lesions in a single prostatectomy specimen. In addition, these EPE-positive lesions demonstrated maximum tumor length and maximum percentage of cancer tissue in the biopsy cores.

The histopathological features of the detected EPEpositive cancers are summarized in Table 4 . In these 14 cancers, the overall Gleason scores of prostatectomy specimens corresponded to those of biopsy specimens. Biopsy specimen parameters relevant to detection are summarized in Table 5. The univariate analysis demonstrated that the maximum percentage of cancer tissue in affected cores was significantly correlated with the presence of EPE $(P=$ 0.022 ), whereas the preoperative serum PSA values, number of positive cores, Gleason score, and maximum tumor length were not $(P=0.25, P=0.16, P=0.14, P=0.091$, respectively). However, no statistically significant factors predicting the presence of EPE were detected among any of the preoperative factors evaluated in this study.

\section{Discussion}

In our present study, 253 individual foci of adenocarcinoma were detected in 93 prostatectomy specimens, with $81 \%$ of the patients having multifocal carcinoma lesions. These results were consistent with those reported in previ-

Table 2. Histopathological features of detected and undetected cancers.

\begin{tabular}{|c|c|c|c|}
\hline & $\begin{array}{l}\text { Detected cancer } \\
\quad(n=168)\end{array}$ & $\begin{array}{l}\text { Undetected cancer } \\
\qquad(n=85)\end{array}$ & $P$ \\
\hline Max. tumor length, mean (mm) & 12.4 & 5.5 & 0.0011 \\
\hline \multicolumn{4}{|l|}{ Localization of tumor } \\
\hline ant & $71(42.3 \%)$ & $39(45.9 \%)$ & 0.81 \\
\hline post & $84(50.0 \%)$ & $41(48.2 \%)$ & \\
\hline ant + post & $13(7.7 \%)$ & $5(5.9 \%)$ & \\
\hline $\mathrm{PZ}$ & $129(76.8 \%)$ & $60(70.6 \%)$ & 0.28 \\
\hline $\mathrm{TZ}$ & $39(23.2 \%)$ & $25(29.4 \%)$ & \\
\hline \multicolumn{4}{|l|}{ Gleason score } \\
\hline 6 & $67(39.9 \%)$ & $64(75.3 \%)$ & $<0.0001$ \\
\hline 7 or more & $101(60.1 \%)$ & $21(24.7 \%)$ & \\
\hline EPE positive & $14(8.3 \%)$ & $1(1.2 \%)$ & $<0.0001$ \\
\hline RM positive & $27(16.1 \%)$ & $0(0 \%)$ & 0.02 \\
\hline pn positive & $55(23.7 \%)$ & $6(7.6 \%)$ & $<0.0001$ \\
\hline
\end{tabular}


Table 3. Association of histopathological features of detected cancers with EPE.

\begin{tabular}{lccc}
\hline & $\begin{array}{c}\text { EPE positive } \\
(n=14)\end{array}$ & $\begin{array}{c}\text { EPE negative } \\
(n=154)\end{array}$ & $P$ \\
\hline Biopsy results, mean & & & $\mathbf{0 . 0 1}$ \\
No. of positive cores & $2.9 \pm 1.4$ & $1.8 \pm 1.4$ & $\mathbf{0 . 0 0 0 3}$ \\
Max. \%cancer (\%) & $46.4 \pm 26.9$ & $24.5 \pm 20.6$ & $<\mathbf{0 . 0 0 0 1}$ \\
Max. tumor length (mm) & $6.6 \pm 3.1$ & $3.5 \pm 2.9$ & \\
Prostatectomy specimen $(\%)$ & & & $\mathbf{0 . 0 0 7}$ \\
Localization of tumor & $12(85.7 \%)$ & $70(45.5 \%)$ & \\
$\quad$ ant & $1(7.1 \%)$ & $72(46.8 \%)$ & 0.1 \\
post & $13(92.9 \%)$ & $12(7.8 \%)$ & \\
ant + post & $1(7.1 \%)$ & $38(24.7 \%)$ & \\
PZ & & & $\mathbf{0 . 0 0 9}$ \\
TZ & $1(7.1 \%)$ & $66(42.9 \%)$ & \\
Gleason score & $13(92.9 \%)$ & $88(57.1 \%)$ & 0.6 \\
6 & $3(21.4 \%)$ & $24(15.6 \%)$ & $<\mathbf{0 . 0 0 0 1}$ \\
7 or more & $13(92.9 \%)$ & $48(31.2 \%)$ & \\
RM & & & \\
pn & $12 \%)$ & \\
\hline
\end{tabular}

Table 4. Histopathological features of EPE-positive, detected cancers (14 cases).

\begin{tabular}{ccc}
\hline Gls of prostatectomy & Gls of biopsy specimen & Max. length of cancer (mm) \\
\hline $3+3$ & $3+3$ & 21 \\
$4+3$ & $4+3$ & 20 \\
$4+4$ & $4+4$ & 16 \\
$3+4$ & $3+4$ & 17 \\
$3+4$ & $3+4$ & 19 \\
$3+4$ & $3+4$ & 22 \\
$4+3$ & $4+3$ & 20 \\
$4+3$ & $4+3$ & 20 \\
$4+3$ & $3+4$ & 18 \\
$4+3$ & $4+3$ & 15 \\
$3+4$ & $3+3$ & 16 \\
$4+3$ & $3+4$ & 20 \\
$4+3$ & $4+3$ & 19 \\
$4+3$ & $4+3$ & 17 \\
\hline
\end{tabular}

Table 5. Predictive value of preoperative parameters for EPE.

\begin{tabular}{lcc}
\hline \multicolumn{1}{c}{ Variables } & $\begin{array}{c}\text { Univariate analysis } \\
(P \text { values })\end{array}$ & $\begin{array}{c}\text { Multivariate analysis } \\
(P \text { values })\end{array}$ \\
\hline Serum PSA & 0.25 & 0.30 \\
No. of positive cores & 0.16 & 0.62 \\
Max. tumor length $(\mathrm{mm})$ & 0.091 & 0.52 \\
Max. \%cancer $(\%)$ & $\mathbf{0 . 0 2 2}$ & 0.14 \\
Biopsy Gleason score & 0.14 & 0.17 \\
\hline
\end{tabular}

ous studies (Arora et al. 2004; Cheng et al. 2005; Andreoiu and Cheng 2010). Meticulous marking by black ink to identify the anterior and posterior side of prostate core needle biopsies, thereby identifying the anterior and posterior ends of the specimen, enabled precise localization of the cancer prior to surgery, resulting in appropriate selection of surgical procedures. Thus, we could identify the precise intraprostatic lesion site for $66.4 \%$ (168 cancer lesions of 
253 total cancers) of the lesions. This marking technique, although somewhat time-consuming and labor-intensive, may substantively contribute to optimizing the selection of surgical techniques for prostatectomy. Meeks et al. (2013) also reported the difference in cancer detectability by biopsy according to the site of the cancer, in which they divided the prostate gland into six regions (basal, middle, and apex portion in each left and right lobe) and reported that the detection rates of carcinoma in prostate biopsies varied from $67 \%$ to $82 \%$ in the different regions of the prostate. However, to the best of our knowledge, there have been no previous reports on the detection of carcinoma sites in prostatectomy specimens retrieved by preoperative prostate biopsy. The detection rate of the carcinoma from the biopsy specimens was $64 \%$, which appeared to be relatively low. We speculate that following were the reasons underlying this relatively low detection rate: 1) multiple foci of adenocarcinoma existed in a single cutting slice, so there were many small cancer foci that went undetected and were not included in the biopsy cores; 2) conversely, a single cancer focus, especially large-sized lesions, could be detected in two or more biopsy cores.

We performed all biopsies using the transrectal approach, which makes it easier to detect cancers located in the PZ than in the TZ. However, there were no significant differences in terms of preoperative detectability between cancers located in the PZ and the TZ in the present study, although a slightly higher detection rate of lesions in the PZ was observed. This may be because of both the good detectability of TZ cancers using our biopsy methods and the presence of many undetected small cancers in the PZ region; however, further investigations are warranted for clarification.

In the present study, preoperative cancer detectability was significantly associated with the tumor size, Gleason score, and EPE status by prostatectomy, i.e., preoperatively detectable cancer lesions were significantly larger and had significantly higher Gleason scores and EPE positivity than undetectable lesions. These results also indicated that clinically important cancer lesions could be detected using our systematic 12-core biopsy method, and many undetected cancers could be of minor clinical importance. The factor most significantly influencing cancer detectability was the size of the cancer and not the Gleason score or EPE presence.

The results of our study regarding the prediction of the EPE status in operative specimens were also consistent with those observed in previous studies, in which factors predicting EPE in biopsy specimens were reported as the number of corresponding positive cores, maximum tumor length, maximum cancer-involvement percentage among corresponding cores, and Gleason score (Pound et al. 1999; Stamey et al. 1999; Quinn et al. 2003; Ishizaki et al. 2012). In addition, we demonstrated a significant association of these parameters in the preoperative biopsy with the EPE status in the operated prostatectomy specimen for the first time using cancer site prediction methods based on the biopsy procedure with the marking technique. In addition, we also demonstrated that the relative percentage of cancer tissue throughout the biopsy length was the only statistically significant factor in the univariate analysis. This could be due to the relatively higher number of EPEpositive patients included in this study compared with previous studies (Gao et al. 2000; Ohori et al. 2004; Yamanaka et al. 2013); however, further investigations are required for clarification.

This study also indicated the usefulness of cancer-site prediction by the biopsy procedure that included marking of the anterior and posterior ends of each biopsy core, as previously reported (Galosi et al. 2011; Yamanaka et al. 2013). Yamanaka et al. (2013) demonstrated by multivariate analysis that the presence of cancer on the posterior margin of the biopsy core was one of the significant independent predictors for EPE. In this study, we also demonstrated that the EPE-positive cancers were observed in biopsy cores with maximum tumor lengths and maximum cancerinvolvement percentage. The overall predictive rate of the EPE status among the preoperative biopsy factors was relatively low, but the possibility of the presence of EPE should be suspected in preoperative biopsies with the largest percentage of cancer tissue. By combining cancer-site prediction methods, the accurate position of the EPE-positive cancer can be achieved preoperatively, leading to appropriate decision-making regarding the surgical technique. Marking with black ink as reported in this study could provide critical information and is by no means time-consuming or labor intensive.

With this simple but novel marking method of surgical biopsy specimens, we could identify the exact intraprostatic location of the lesions from which biopsy specimens were obtained. The biopsy method that we introduced in this particular study enables relevant selection of surgical procedures on an individual basis.

\section{Conclusion}

For the first time, we could compare the histopathological factors precisely between preoperative and operative biopsy specimens using cancer-site prediction methods and identified the factors associated with the EPE status. Cancer-site prediction by preoperative biopsies systematically obtained by 12-core needles, marking, and precise identification of the anterior-posterior site as well as measurement of cancer volume proved clinically critical to selecting the appropriate operative strategy.

\section{Conflict of Interest}

The authors declare no conflict of interest.

\section{References}

Andreoiu, M. \& Cheng, L. (2010) Multifocal prostate cancer: biologic, prognostic, and therapeutic implications. Hum. Pathol., 41, 781-793. 
Arora, R., Koch, M.O., Eble, J.N., Ulbright, T.M., Li, L. \& Cheng, L. (2004) Heterogeneity of Gleason grade in multifocal adenocarcinoma of the prostate. Cancer, 100, 2362-2366.

Babaian, R.J., Toi, A., Kamoi, K., Troncoso, P., Sweet, J., Evans, R., Johnston, D. \& Chen, M. (2000) A comparative analysis of sextant and an extended 11-core multisite directed biopsy strategy. J. Urol., 163, 152-157.

Bjurlin, M.A., Carter, H.B., Schellhammer, P., Cookson, M.S., Gomella, L.G., Troyer, D., Wheeler, T.M., Schlossberg, S., Penson, D.F. \& Taneja, S.S. (2013) Optimization of initial prostate biopsy in clinical practice: sampling, labeling and specimen processing. J. Urol., 189, 2039-2046.

Bostwick, D.G. (1994) Grading prostate cancer. Am. J. Clin. Pathol., 102, S38-56.

Cheng, L., Jones, T.D., Pan, C.X., Barbarin, A., Eble, J.N. \& Koch, M.O. (2005) Anatomic distribution and pathologic characterization of small-volume prostate cancer $(<0.5 \mathrm{ml})$ in wholemount prostatectomy specimens. Mod. Pathol., 18, 10221026.

Eskew, L.A., Bare, R.L. \& McCullough, D.L. (1997) Systematic 5 region prostate biopsy is superior to sextant method for diagnosing carcinoma of the prostate. J. Urol., 157, 199-202; discussion 202-203.

Firoozi, F., Nazeer, T., Fisher, H.A., Kaufman, R.P. Jr., White, M.D. \& Mian, B.M. (2009) Tissue-marking scheme for a cost-effective extended prostate biopsy protocol. Urol. Oncol., 27, 21-25.

Galosi, A.B., Muzzonigro, G., Lacetera, V. \& Mazzucchelli, R. (2011) Specimen orientation by marking the peripheral end: (potential) clinical advantages in prostate biopsy. Prostate Cancer, 2011, 270403.

Gao, X., Mohideen, N., Flanigan, R.C., Waters, W.B., Wojcik, E.M. \& Leman, C.R. (2000) The extent of biopsy involvement as an independent predictor of extraprostatic extension and surgical margin status in low risk prostate cancer: implications for treatment selection. J. Urol., 164, 1982-1986.

Ishizaki, F., Hara, N., Koike, H., Kawaguchi, M., Tadokoro, A., Takizawa, I., Nishiyama, T., Takahashi, K. \& Hohenfellner, R. (2012) Prediction of pathological and oncological outcomes based on extended prostate biopsy results in patients with prostate cancer receiving radical prostatectomy: a single institution study. Diagn. Pathol., 7, 68.

Meeks, J.J., Walker, M., Bernstein, M., Kent, M. \& Eastham, J.A. (2013) Accuracy of post-radiotherapy biopsy before salvage radical prostatectomy. BJU Int., 112, 308-312.

Ohori, M., Kattan, M.W., Koh, H., Maru, N., Slawin, K.M., Shariat, S., Muramoto, M., Reuter, V.E., Wheeler, T.M. \& Scardino, P.T. (2004) Predicting the presence and side of extracapsular extension: a nomogram for staging prostate cancer. J. Urol., 171, 1844-1849.

Orikasa, K., Ito, A., Ishidoya, S., Saito, S., Endo, M. \& Arai, Y. (2008) Anterior apical biopsy: is it useful for prostate cancer detection? Int. J. Urol., 15, 900-904; discussion 904.

Pound, C.R., Partin, A.W., Eisenberger, M.A., Chan, D.W., Pearson, J.D. \& Walsh, P.C. (1999) Natural history of progression after PSA elevation following radical prostatectomy. JAMA, 281, 1591-1597.

Quinn, D.I., Henshall, S.M., Brenner, P.C., Kooner, R., Golovsky, D., O’Neill, G.F., Turner, J.J., Delprado, W., Grygiel, J.J., Sutherland, R.L. \& Stricker, P.D. (2003) Prognostic significance of preoperative factors in localized prostate carcinoma treated with radical prostatectomy: importance of percentage of biopsies that contain tumor and the presence of biopsy perineural invasion. Cancer, 97, 1884-1893.

Sonoda, T., Nagata, Y., Mori, M., Miyanaga, N., Takashima, N., Okumura, K., Goto, K., Naito, S., Fujimoto, K., Hirao, Y., Takahashi, A., Tsukamoto, T., Fujioka, T. \& Akaza, H. (2004) A case-control study of diet and prostate cancer in Japan: possible protective effect of traditional Japanese diet. Cancer Sci., 95, 238-242.

Stamey, T.A., McNeal, J.E., Yemoto, C.M., Sigal, B.M. \& Johnstone, I.M. (1999) Biological determinants of cancer progression in men with prostate cancer. JAMA, 281, 1395-1400.

Terris, M.K. \& McNeal, J.E. (2002) Application of tissue-marking ink to prostate biopsy specimens. Prostate, 50, 247-251.

Yamanaka, H., Matsumoto, K., Obata, J., Ninomiya, A., Mukai, K. \& Nakamura, S. (2013) Positive posterior margin of needle biopsy cores is an independent predictor for extracapsular extension in retropubic radical prostatectomy. Urology, 81, 986-989. 